\title{
B-SPLINE COLLOCATION SOLUTION FOR AN EQUATION ARISING IN INSTABILITY PHENOMENON
}

\author{
H. V. Chapani ${ }^{1}$ \\ ${ }^{1}$ Assistant Professor, Department of Mathematics, Govt. College of Arts, Commerce and Science, Silvassa, Dadra and \\ Nagar Haveli, India
}

\begin{abstract}
In the present paper numerical discusses a theoretical model for instability phenomenon in double phase flow through homogeneous porous medium. Relation between relative permeability and saturation has been considered based on earlier experiment. A governing nonlinear partial differential equation is solved by collocation method with cubic B-splines. To obtain the scheme of the equation the nonlinear term is approximated by Taylor series which leads to tridiagonal system and has been solved by well-known Thomas Algorithm. The Numerical solution is obtained by using MATLAB coding.
\end{abstract}

Keywords: Darcy's law, Taylor series, Saturation, Cubic B-splines

\section{INTRODUCTION}

In oil recovery technology it is common practice to inject water into the oil field at certain spots in an attempt to drive oil to other spots for pumping [1]. When fluid is displaced by a lesser viscous fluid in porous media due to the injection, instead of regular displacement of whole front, protuberance takes place and shoot the medium at relatively high speed. This phenomenon is called fingering or instability.

Many researchers discussed this phenomenon analytically/numerically with different point of view. Verma [2] examined fingering behavior by statistical viewpoints in immiscible displacement through heterogeneous porous media with assumption of constant mean pressure of injected fluid and native fluid. He obtained the analytical solution of the problem by analytical method. Scheidegger [3] neglected the size and shape of individual fingers and considered only the average cross sectional area of fingers occupied in porous media. Scheidegger and Johnson [4] analyzed the growth of instabilities in homogeneous porous media without capillary pressure. Some of the researchers analyzed the phenomenon with numerical method. Recently Pradhan et. all [5] discussed the numerical solution of the phenomenon by Finite Element Technique. Borana et. all [6] obtained the numerical solution of the instability phenomenon using Crank-Nicolson Finite Difference Method. They considered the linear relationship between relative permeability of the injecting fluid and saturation of injecting fluid.

In the present paper problem of fingering in homogeneous porous medium with capillary pressure from the statistical view point has been discussed. Homogeneous porous matrix has been considered for analysis of the problem and assumed that the Darcy-like formulation is sufficient for the flow equations. Specific relation between relative permeability and phase saturation have been considered from the experiment based analysis. The mathematical formulation of the problem yields a non-linear second order time dependent partial differential equation which has been solved by applying B-spline finite element method with specific initial and boundary conditions. The graphical representation of the solution is given as graph of saturation of injected fluid verses time and saturation of injected fluid verses distance.

\section{STATEMENT OF THE PROBLEM}

Consider a uniform injection of less viscous fluid into a more viscous fluid in a saturated homogeneous porous medium with length L. Here injected fluid shoots the native fluid and protuberances takes place, this develops fingers at the initial boundary $\mathrm{x}=0$ ( $\mathrm{x}$ is measured in the displacement direction). Further, at the initial boundary, it is assumed that the complete saturation exist. The problem is to determine the equation of saturation describing the instability phenomenon which is shown in Fig.-1.

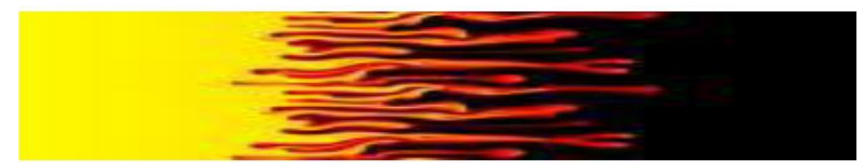

Fig.-1: Instability Phenomenon

\section{MATHEMATICAL FORMULATION OF THE}

\section{PROBLEM}

By Darcy's law the velocity of injected fluid $\left(v_{i}\right)$ and native fluid $\left(v_{n}\right)$ can be expressed as [7]

$$
v_{i}=-\frac{k_{i}}{\mu_{i}} k\left(\frac{\partial p_{i}}{\partial x}\right),
$$


$v_{n}=-\frac{k_{n}}{\mu_{n}} k\left(\frac{\partial p_{n}}{\partial x}\right)$

where $\mathrm{k}$ is the permeability of porous medium, $k_{i}$ and $k_{n}$ are relative permeabilities of injected fluid and native fluid $p_{i}$ and $p_{n}$ are pressures, $\mu_{i}$ and $\mu_{n}$ are constant kinematic viscosities of native fluid and injected fluid respectively.

Equations of conservation of mass for two immiscible fluids are given by

$\phi \frac{\partial S_{i}}{\partial t}+\frac{\partial v_{i}}{\partial x}=0$

$\phi \frac{\partial S_{n}}{\partial t}+\frac{\partial v_{n}}{\partial x}=0$

where $\phi$ is the porosity of the medium and $S_{i}, S_{n}$ are saturation of injected and native fluid respectively.

By definition of phase saturation, the sum of saturations is equal to 1. i.e.

$S_{i}+S_{n}=1$.

Consider the following experiment based relations [8] for relative permeability

$k_{i}=S_{i}^{a}$

$k_{n}=S_{n}^{b}=\left(1-S_{i}\right)^{b}$

where $1 \leq a, b \leq 3$.

Capillary pressure $p_{c}$ is defined as the difference between pressures $p_{n}$ and $p_{i}$ of two immiscible fluids, i.e.

$p_{c}=p_{n}-p_{i}$.

Hence, capillary pressure gradient relates the pressure gradient of $p_{n}$ and $p_{i}$.

$\frac{\partial p_{c}}{\partial x}=\frac{\partial p_{n}}{\partial x}-\frac{\partial p_{i}}{\partial x}$

Substituting the value of $\frac{\partial p_{i}}{\partial x}$ from equation (8) into the equation (1), we get

$v_{i}=-\frac{k_{i}}{\mu_{i}} k\left(\frac{\partial p_{n}}{\partial x}-\frac{\partial p_{c}}{\partial x}\right)$.
Mehta [9] suggested the following linear relationship between capillary pressure and displacing fluid.

$$
p_{c}=-\beta S_{i}
$$

where $\beta$ is proportionality constant.

If the flow takes place in $\mathrm{x}$ direction only, capillary pressure gradient may be written as

$\frac{\partial p_{c}}{\partial x}=-\beta \frac{\partial S_{i}}{\partial x}$

Using equation (9) and (2) in equations (3) and (4) respectively, we obtain

$\phi \frac{\partial S_{i}}{\partial t}-\frac{\partial}{\partial x}\left(\frac{k_{i}}{\mu_{i}} k\left(\frac{\partial p_{n}}{\partial x}-\frac{\partial p_{c}}{\partial x}\right)\right)=0$

$\phi \frac{\partial S_{n}}{\partial t}-\frac{\partial}{\partial x}\left(\frac{k_{n}}{\mu_{n}} k \frac{\partial p_{n}}{\partial x}\right)=0$

Combining equation (11) and (12) and using equation (5), we have

$\frac{\partial}{\partial x}\left[\left(\frac{k_{i}}{\mu_{i}} k+\frac{k_{n}}{\mu_{n}} k\right) \frac{\partial p_{n}}{\partial x}-\frac{k_{i}}{\mu_{i}} k \frac{\partial p_{c}}{\partial x}\right]=0$

Integrating equation (13) with respect to $\mathrm{x}$ gives

$\left(\frac{k_{i}}{\mu_{i}} k+\frac{k_{n}}{\mu_{n}} k\right) \frac{\partial p_{n}}{\partial x}-\frac{k_{i}}{\mu_{i}} k \frac{\partial p_{c}}{\partial x}=-K_{*}$,

where $K_{*}$ is a constant of integration.

i.e. $\frac{\partial p_{n}}{\partial x}=\frac{-K_{*}}{\frac{k_{i}}{\mu_{i}} k\left(1+\frac{k_{n} \mu_{i}}{k_{i} \mu_{n}}\right)}+\frac{\frac{\partial p_{c}}{\partial x}}{\left(1+\frac{k_{n} \mu_{i}}{k_{i} \mu_{n}}\right)}$

Hence equation (11) takes the form

$\phi \frac{\partial S_{i}}{\partial t}+\frac{\partial}{\partial x}\left(\frac{k \frac{k_{n}}{\mu_{n}} \frac{\partial p_{c}}{\partial x}}{1+\frac{k_{n} \mu_{i}}{k_{i} \mu_{n}}}+\frac{K_{*}}{1+\frac{k_{n} \mu_{i}}{k_{i} \mu_{n}}}\right)=0$.

We can write the pressure of native fluid $p_{n}$ as

$p_{n}=\frac{p_{i}+p_{n}}{2}+\frac{p_{i}-p_{n}}{2}$ 
Thus using mean pressure $\bar{P}=\frac{p_{i}+p_{n}}{2}$ and equation (7) in equation (17), pressure of native fluid can be expressed as

$p_{n}=\bar{P}+\frac{p_{c}}{2}$

Here we note that the mean pressure is constant [2] and so hence

$\frac{\partial p_{n}}{\partial x}=\frac{1}{2} \frac{\partial p_{c}}{\partial x}$

Substituting the value of $\frac{\partial p_{n}}{\partial x}$ from the equation (19) into the equation (14) gives value of integration constant $K_{*}$ as

$K_{*}=\frac{1}{2}\left(\frac{k_{i}}{\mu_{i}} k-\frac{k_{n}}{\mu_{n}} k\right) \frac{\partial p_{c}}{\partial x}$

Substituting the value of $K_{*}$ from the equation (20) into the equation (16) gives

$\phi \frac{\partial S_{i}}{\partial t}+\frac{1}{2} \frac{\partial}{\partial x}\left(k \frac{k_{i}}{\mu_{i}} \frac{\partial p_{c}}{\partial x}\right)=0$

Using the linear relation between capillary pressure and saturation of injected fluid, equation (21) may be written as

$\phi \frac{\partial S_{i}}{\partial t}+\frac{1}{2} \frac{\partial}{\partial x}\left(k \frac{k_{i}}{\mu_{i}} \frac{\partial p_{c}}{\partial S_{i}} \frac{\partial S_{i}}{\partial x}\right)=0$

From equation (6a) and (10b), equation (22) takes the form

$\phi \frac{\partial S_{i}}{\partial t}=\frac{k}{2 \mu_{i}} \frac{\partial}{\partial x}\left(\beta S_{i}^{a} \frac{\partial S_{i}}{\partial x}\right)$

Suitable boundary and initial conditions are taken as

$S_{i}(0, t)=S_{i 0} \quad$ at $\quad x=0$ and $t>0$

$\frac{\partial S_{i}}{\partial x}(L, t)=S_{i L} \quad$ at $\quad x=L$ and $t>0$

$S_{i}(x, 0)=S_{0}$ at $t=0$ and $x>0$

Equation (23) the governing equation describing instability phenomenon in double phase flow through porous media together with boundary and initial condition in equation (24a), (24b) and (25) respectively.
Introduce the following dimensionless variables

$X=\frac{x}{L}, T=\frac{\beta k t}{2 \phi L^{2} \mu_{i}}$

where $0 \leq X \leq 1$.

Then the dimensionless form of the equation (23) is given by

$$
\frac{\partial S_{i}}{\partial T}=\frac{\partial}{\partial X}\left(S_{i}^{a} \frac{\partial S_{i}}{\partial X}\right)
$$

The boundary and initial conditions in the form of dimensionless variables are as under:

$$
\begin{aligned}
& S_{i}(0, T)=S_{i 0} \quad(T>0) \\
& \frac{\partial S_{i}}{\partial X}(1, T)=S_{i L} \quad(T>0) \\
& S_{i}(X, 0)=S_{0} \quad 0 \leq X \leq 1
\end{aligned}
$$

The condition (28a) and (28b) represents saturation of injected fluid at $X=0$ and at $x=L$, condition (29) represents initial saturation of injected fluid. For simplicity we replace $S_{i}$ by $\mathrm{S}$.

\section{METHOD OF NUMERICAL SOLUTION}

The interval $[0,1]$ is divided into $\mathrm{N}$ elements with equal length $\Delta X=h$ by the knots $X_{i}$ such that $0=X_{0}<X_{1}<X_{2}<\ldots . .<X_{N}=1$. Cubic B-Splines [10] are defined by

$$
\phi_{m}(X)=\frac{1}{h^{3}}\left\{\begin{array}{lr}
\left(X-X_{m-2}\right)^{3}, & {\left[\mathrm{X}_{m-2}, \mathrm{X}_{m-1}\right],} \\
\left(X-X_{m-2}\right)^{3}-4\left(X-X_{m-1}\right)^{3}, & {\left[\mathrm{X}_{m-1}, \mathrm{X}_{m}\right],} \\
\left(X_{m+2}-X\right)^{3}-4\left(X_{m+1}-X\right)^{3}, & {\left[\mathrm{X}_{m}, \mathrm{X}_{m+1}\right],} \\
\left(X_{m+2}-X\right)^{3}, & {\left[\mathrm{X}_{m+1}, \mathrm{X}_{m+2}\right],} \\
0, & \text { otherwise, }
\end{array}\right.
$$

where $m=-1,0,1 \ldots, N$

Then the set of cubic B-splines $\left\{\phi_{m}\right\}_{m=-1}^{N+1}$ constitutes a basis for the functions defined on $[0,1]$ and the approximate solution $\mathrm{S}(\mathrm{X}, \mathrm{T})$ on $[0,1]$ to the exact solution of the problem is written as

$$
S(X, T)=\sum_{m=-1}^{N+1} \delta_{m}(T) \phi_{m}(X)
$$


where $\delta_{m}(T)$ are time dependent unknowns parameters which are to be determined at each time level.

By definition of cubic B-splines it is evident that each spline covers four consecutive subintervals so that each subinterval covered by four consecutive cubic B-splines. Therefore, at the node $X_{m}$, the value of unknown variable $S_{m}$ and its first and second order derivatives with respect to the space variable can be related by $\delta_{m-1}, \delta_{m}, \delta_{m+1}$.

$$
\begin{aligned}
& S_{m}=\delta_{m-1}+4 \delta_{m}+\delta_{m+1}, \\
& h S_{m}^{\prime}=3\left(\delta_{m+1}-\delta_{m-1}\right), \\
& h^{2} S_{m}^{\prime \prime}=6\left(\delta_{m-1}-2 \delta_{m}+\delta_{m+1}\right),
\end{aligned}
$$

Rewriting equation (27) into the simple form

$$
S_{T}=S^{a} S_{X X}+a S^{a-1}\left(S_{X}\right)^{2}
$$

To solve the equation (32), discretizing the time derivative using forward difference formula and applying $\theta$-weighted $(0 \leq \theta \leq 1)$ scheme to the space derivative the equation

$$
\begin{aligned}
\frac{S^{n+1}-S^{n}}{\Delta T} & -\theta\left\{\left(S^{a} S_{X X}\right)^{n+1}+\left(S^{a} S_{X X}\right)^{n}\right\} \\
& -a(1-\theta)\left\{\left(S^{a-1} S_{X}^{2}\right)^{n+1}+\left(S^{a-1} S_{X}^{2}\right)^{n}\right\}=0
\end{aligned}
$$

where $\Delta T$ is time step and $\mathrm{n}$ and $\mathrm{n}+1$ denote the successive time levels, is obtained.

Letting $\theta=1 / 2$, equation (33) may be written as

$$
\begin{aligned}
\frac{S^{n+1}-S^{n}}{\Delta T} & -\left\{\frac{\left(S^{a} S_{X X}\right)^{n+1}+\left(S^{a} S_{X X}\right)^{n}}{2}\right\} \\
& -a\left\{\frac{\left(S^{a-1} S_{X}^{2}\right)^{n+1}+\left(S^{a-1} S_{X}^{2}\right)^{n}}{2}\right\}=0
\end{aligned}
$$

Using Taylor series the nonlinear terms in equation (34) are linearized by

$$
\begin{aligned}
\left(S^{a} S_{X X}\right)^{n+1}= & a\left(S^{n}\right)^{a-1} S_{X X}^{n} S^{n+1}+\left(S^{n}\right)^{a} S_{X X}^{n+1} \\
& -a\left(S^{n}\right)^{a} S_{X X}^{n} \\
\left(S^{a-1} S_{X}^{2}\right)^{n+1}= & a\left\{\begin{array}{l}
(a-1)\left(S^{n}\right)^{a-2}\left(S_{X}^{n}\right)^{2} S^{n+1}+ \\
2\left(S^{n}\right)^{a-1} S_{X}^{n} S_{X}^{n+1}-a\left(S^{n}\right)^{a-1}\left(S_{X}^{n}\right)^{2}
\end{array}\right\}
\end{aligned}
$$

For the sake of simplicity we use the notations $T_{1}, T_{2}, T_{3}$ for $S_{m}, S_{m}^{\prime}, S_{m}^{\prime \prime}$, respectively, at $n$th time step.

$$
\begin{aligned}
& T_{1}=\delta_{m-1}+4 \delta_{m}+\delta_{m+1}, \\
& T_{2}=\left(\frac{3}{h}\right)\left(\delta_{m+1}-\delta_{m-1}\right), \\
& T_{3}=\left(\frac{6}{h^{2}}\right)\left(\delta_{m-1}-2 \delta_{m}+\delta_{m+1}\right),
\end{aligned}
$$

Using equations (35) and (36) in equation (34) and simplifying, we get

$$
\begin{aligned}
& P \delta_{m-1}^{n+1}+Q \delta_{m}^{n+1}+R \delta_{m+1}^{n+1}=S \\
& P=V_{1}-(3 / h) V_{2}+\left(6 / h^{2}\right) V_{3} ; \\
& \text { where } \begin{array}{l}
Q=4 V_{1}-\left(12 / h^{2}\right) V_{3} ; \\
R=V_{1}+(3 / h) V_{2}+\left(6 / h^{2}\right) V_{3} ; \quad \text { with }
\end{array} \\
& S=2 T_{1}-\Delta T(a-1) T_{1}^{(a-1)}\left(T_{1} T_{3}+a^{2} T_{2}^{2}\right) \text {; } \\
& V_{1}=2-\Delta T a T_{1}^{(a-2)}\left(T_{1} T_{3}+a(a-1) T_{2}^{2}\right) ; \\
& V_{2}=-2 \Delta T a^{2} T_{1}^{(a-1)} T_{2} \text {; } \\
& V_{3}=-\Delta T T_{1}^{a} \text {; }
\end{aligned}
$$

Equation (37) represents the recurrence relation at $X_{m}$, where $X_{m}$ 's are collocation points with $m=0,1,2, \ldots, \mathrm{N}$.

\section{RESULTS AND DISCUSIONS}

The Equation (37) gives the linear system of $\mathrm{N}+1$ equations in $\mathrm{N}+3$ unknowns. After applying two boundary conditions given in equations (27a) and (27b) two unknowns are eliminated and hence the system reduced to $\mathrm{N}+1$ equations in $\mathrm{N}+1$ unknowns. Also due to the cubic spline functions system (37) is tridiagonal and it can be easily solved by a well-known Thomas Algorithm. To compute numerical results $\mathrm{h}=0.001$ and $\Delta T=0.01$ are taken and suitable boundary conditions $S_{i 0}=1, S_{i L}=0$ and initial condition $S_{0}=e^{-X}$ are considered. Equation (27) together with boundary conditions (28a), (28b) and initial condition (29) is recursively solved for fixed value of $a$. Numerical values of saturation of injecting fluid are presented in Table 1- 3 for $a=1$ - 3 respectively. Fig. 2 - 4 show the graphical representation of the saturation for various dimensionless time and Fig. 5-7 represent the saturation for dimensionless distance. From Fig. 2 - 4 saturation of injected fluid decreases with distance and from Fig. 5 - 7 saturation of injected fluid increases with time.

Table- 1: Saturation of injected fluid when $a=1$

\begin{tabular}{|l|l|l|l|l|l|}
\hline $\mathrm{X} / \mathrm{T}$ & 0.2000 & 0.4000 & 0.6000 & 0.8000 & 1.0000 \\
\hline 0.0000 & 1.0000 & 1.0000 & 1.0000 & 1.0000 & 1.0000 \\
\hline 0.1000 & 0.9496 & 0.9646 & 0.9763 & 0.9847 & 0.9903 \\
\hline 0.2000 & 0.8973 & 0.9286 & 0.9525 & 0.9694 & 0.9807 \\
\hline 0.3000 & 0.8440 & 0.8927 & 0.9292 & 0.9547 & 0.9715 \\
\hline 0.4000 & 0.7906 & 0.8577 & 0.9070 & 0.9408 & 0.9630 \\
\hline
\end{tabular}


\begin{tabular}{|l|l|l|l|l|l|}
0.5000 & 0.7387 & 0.8248 & 0.8865 & 0.9282 & 0.9552 \\
\hline
\end{tabular} \begin{tabular}{|l|l|l|l|l|l|}
0.6000 & 0.6902 & 0.7951 & 0.8685 & 0.9173 & 0.9485 \\
\hline
\end{tabular} \begin{tabular}{|l|l|l|l|l|l|l|}
0.7000 & 0.6476 & 0.7700 & 0.8536 & 0.9083 & 0.9431 \\
\hline 0.8000 & 0.6138 & 0.7509 & 0.8425 & 0.9017 & 0.9391 \\
\hline
\end{tabular} \begin{tabular}{|l|l|l|l|l|l|}
\hline 0.8000 & 0.6138 & 0.7509 & 0.8425 & 0.9017 & 0.9391 \\
\hline
\end{tabular} \begin{tabular}{|l|l|l|l|l|l|}
0.9000 & 0.5920 & 0.7388 & 0.8356 & 0.8976 & 0.9367 \\
\hline
\end{tabular} \begin{tabular}{|l|l|l|l|l|l|l|}
1.0000 & 0.5844 & 0.7347 & 0.8333 & 0.8962 & 0.9358 \\
\hline
\end{tabular}

Table- 2: Saturation of injected fluid when $a=2$ \begin{tabular}{|l|l|l|l|l|l|}
\hline $\mathrm{X} \backslash \mathrm{T}$ & 0.2000 & 0.4000 & 0.6000 & 0.8000 & 1.0000 \\
\hline
\end{tabular} \begin{tabular}{|l|l|l|l|l|l|l|}
\hline 0.0000 & 1.0000 & 1.0000 & 1.0000 & 1.0000 & 1.0000 \\
\hline
\end{tabular} \begin{tabular}{|l|l|l|l|l|l|}
0.1000 & 0.9702 & 0.9786 & 0.9852 & 0.9902 & 0.9938 \\
\hline
\end{tabular} \begin{tabular}{|l|l|l|l|l|l|}
\hline 0.2000 & 0.9369 & 0.9556 & 0.9697 & 0.9803 & 0.9875 \\
\hline
\end{tabular} \begin{tabular}{|l|l|l|l|l|l|}
0.3000 & 0.8998 & 0.9310 & 0.9539 & 0.9704 & 0.9814 \\
\hline
\end{tabular} \begin{tabular}{|l|l|l|l|l|l|}
0.4000 & 0.8586 & 0.9053 & 0.9381 & 0.9609 & 0.9757 \\
\hline
\end{tabular} \begin{tabular}{|l|l|l|l|l|l|}
\hline 0.5000 & 0.8131 & 0.8788 & 0.9228 & 0.9520 & 0.9704 \\
\hline
\end{tabular} \begin{tabular}{|l|l|l|l|l|l|}
0.6000 & 0.7634 & 0.8526 & 0.9088 & 0.9440 & 0.9657 \\
\hline
\end{tabular} \begin{tabular}{ll|l|l|l|l|l|}
0.7000 & 0.7102 & 0.8281 & 0.8966 & 0.9374 & 0.9619 \\
\hline
\end{tabular} \begin{tabular}{|l|l|l|l|l|l|}
0.8000 & 0.6567 & 0.8076 & 0.8872 & 0.9324 & 0.9591 \\
\hline
\end{tabular} \begin{tabular}{|l|l|l|l|l|l|}
0.9000 & 0.6119 & 0.7936 & 0.8812 & 0.9292 & 0.9573 \\
\hline
\end{tabular} \begin{tabular}{|l|l|l|l|l|l|}
1.0000 & 0.5931 & 0.7887 & 0.8791 & 0.9282 & 0.9567 \\
\hline
\end{tabular}

Table- 3: Saturation of injected fluid when $a=3$ \begin{tabular}{|l|l|l|l|l|l|l|}
\hline $\mathrm{X} \backslash \mathrm{T}$ & 0.2000 & 0.4000 & 0.6000 & 0.8000 & 1.0000 \\
\hline
\end{tabular} \begin{tabular}{|l|l|l|l|l|l|}
\hline 0.0000 & 1.0000 & 1.0000 & 1.0000 & 1.0000 & 1.0000 \\
\hline
\end{tabular} \begin{tabular}{|l|l|l|l|l|l|}
0.1000 & 0.9839 & 0.9890 & 0.9926 & 0.9952 & 0.9970 \\
\hline
\end{tabular} \begin{tabular}{|l|l|l|l|l|l|}
0.2000 & 0.9654 & 0.9770 & 0.9848 & 0.9904 & 0.9940 \\
\hline
\end{tabular} \begin{tabular}{|l|l|l|l|l|l|l|}
\hline 0.3000 & 0.9443 & 0.9640 & 0.9769 & 0.9856 & 0.9911 \\
\hline
\end{tabular} \begin{tabular}{l|l|l|l|l|l|}
0.4000 & 0.9201 & 0.9502 & 0.9689 & 0.9809 & 0.9883 \\
\hline
\end{tabular} \begin{tabular}{|l|l|l|l|l|l|}
0.5000 & 0.8923 & 0.9359 & 0.9612 & 0.9765 & 0.9858 \\
\hline
\end{tabular} \begin{tabular}{ll|l|l|l|l|l|}
0.6000 & 0.8603 & 0.9215 & 0.9540 & 0.9726 & 0.9835 \\
\hline
\end{tabular} \begin{tabular}{|l|l|l|l|l|l|}
\hline 0.7000 & 0.8236 & 0.9080 & 0.9478 & 0.9693 & 0.9817 \\
\hline
\end{tabular} \begin{tabular}{|l|l|l|l|l|l|l|}
\hline 0.8000 & 0.7821 & 0.8965 & 0.9430 & 0.9669 & 0.9803 \\
\hline
\end{tabular} \begin{tabular}{|l|l|l|l|l|l|}
0.9000 & 0.7409 & 0.8886 & 0.9399 & 0.9653 & 0.9795 \\
\hline
\end{tabular} \begin{tabular}{|l|l|l|l|l|l|}
1.0000 & 0.7203 & 0.8858 & 0.9389 & 0.9648 & 0.9792 \\
\hline
\end{tabular}

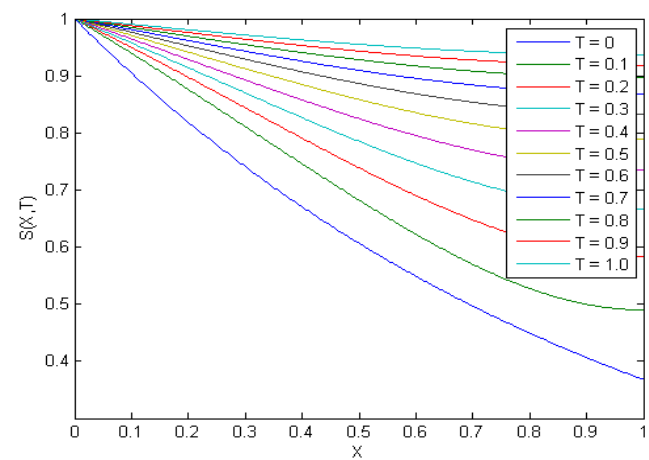

Fig- 2: Saturation of injected fluid for various dimensionless times when $a=1$

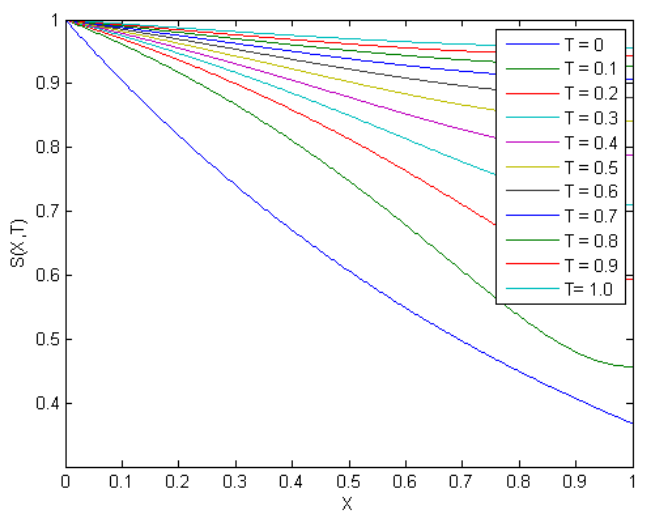

Fig- 3: Saturation of injected fluid for various dimensionless times when $a=2$

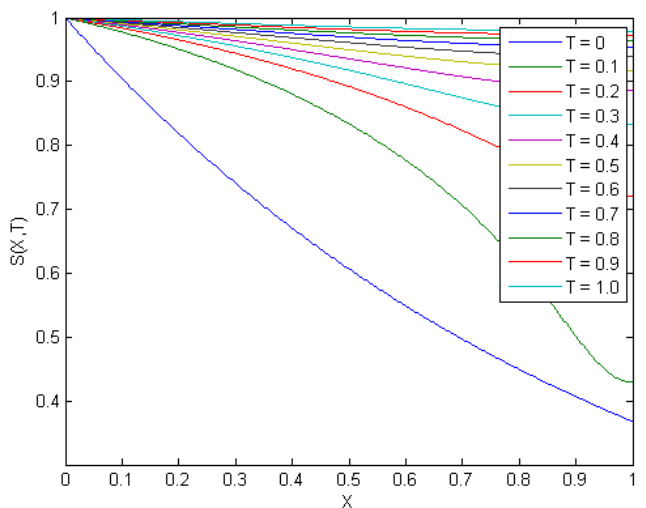

Fig- 4: Saturation of injected fluid for various dimensionless times when $a=3$

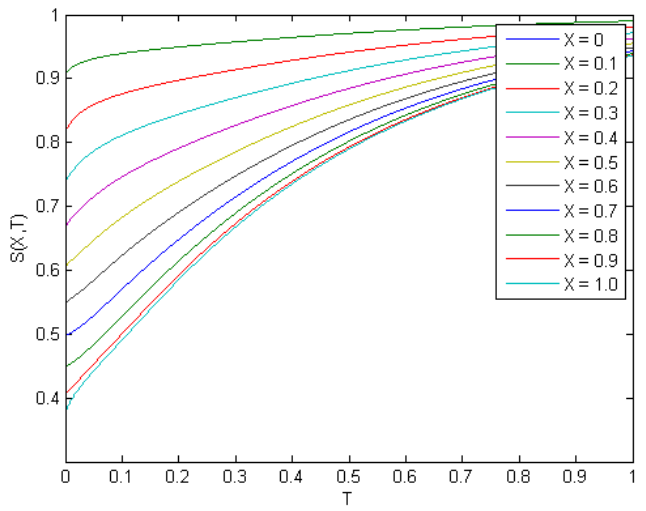

Fig- 5: Saturation of injected fluid for various dimensionless distances when $a=1$ 


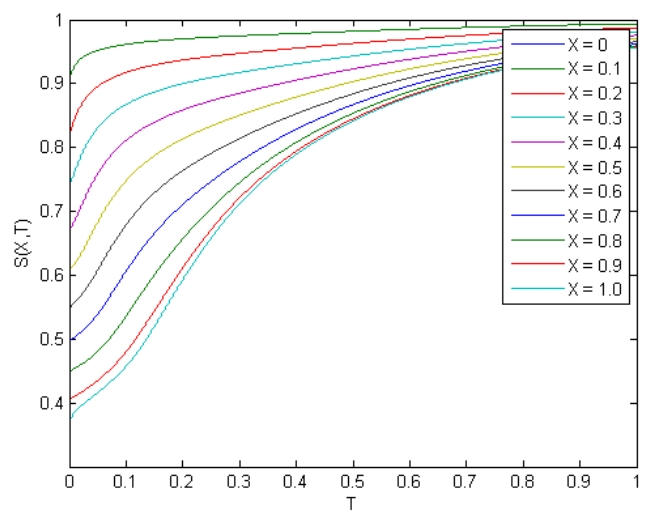

Fig- 6: Saturation of injected fluid for various dimensionless distances when $a=2$

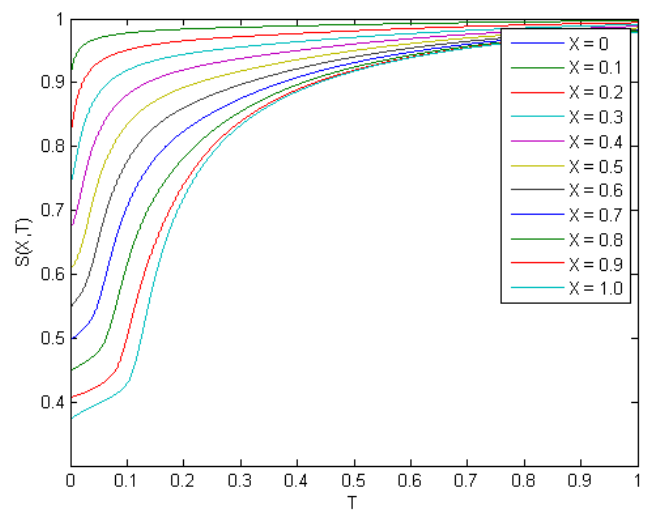

Fig- 7: Saturation of injected fluid for various dimensionless distances when $a=3$

\section{CONCLUSION}

In the present paper one dimensional second order time dependent partial differential equation arising in instability phenomenon has been solved by Cubic B-spline Collocation method. This method can equally apply for solving higher order partial differential equations. Cubic Splines provides second order smooth solution. Saturation of injected fluid decreases with distance and increases with time. Further, it can be concluded that for any fixed distance and fixed time, the saturation of injected fluid increases by increasing value of $a$. This fact is consistent and hence the numerical results resemble the physical phenomenon of the problem.

\section{REFERENCES}

[1]. Brailovsky I., Babchin A., Frankel M., Sivashinsky G., A reduced model for fingering instability in miscible displacement, Physics Letters A, Vol-369, Issue-3, Elsevier, 2007

[2]. Verma A. P., Statistical behavior of fingering in a displacement process in heterogeneous porous medium with capillary pressure, Canadian Journal of Physics, Vol-47, Issue-3, 1969

[3]. Scheidegger A. E., Growth of Instabilities on displacement fronts in Porous Media, Physics of Fluids, Vol-3, 1960
[4]. Scheidegger A. E. and Johnson E. F, The Statistical behavior of Instabilities in displacement process in porous media, Canadian Journal of Physics, 1961

[5]. Pradhan V. H., Mehta M. N., Patel T., A numerical solution of nonlinear equation representing one dimensional instability phenomen in porous media by finite element technique, International Journal of Advanced Engineering Technology, Vol-2, Issue-1,2011

[6]. Borana R. N., Pradhan V. H., Mehta M. N., A solution of instability phenomenon arising in homogeneous porous media by Crank-Nicolson Finite Difference Method, International Journal of Innovative Research in Science, Engineering and Technology, Vol-3, Issue-2, 2014

[7]. Bear J., Dynamics of fluids in porous media, Elsevier, New York, 1972

[8]. Zhang J., Chavent G., Jaffre J., Estimating nonlinearities in two phase flow in porous media, 2009

[9]. Mehta M. N., Asymptotic expansion of fluid flow through porous media, Ph.D. Thesis, South Gujarat University, Surat, India, 1977

[10]. Prenter P.M., Splines and variational methods, New York: Wiley, 1975

\section{BIOGRAPHY}

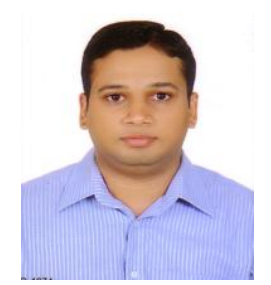

Himanshu V. Chapani received the B.Sc. degree in Mathematics with Gold medal in 2006 and M.Sc. in 2008 from Veer Narmad South Gujarat University, Surat. He also qualified NET with JRF and received Ph.D. Degree from S. V. National institute of Technology, Surat in 2014 under the guidance of Dr. V. H. Pradhan and Prof. M. N. Mehta and has 5 years of teaching experience. His research interests include fluid flow through porous media and finite element methods with B-splines. He is currently working as an Assistant Professor at Govt. College of Arts, Commerce and Science, Silvassa. 